\title{
Caracterização da bacia do rio Japaratuba em Sergipe com auxílio de geotecnologias
}

\author{
Marcus Aurélio Soares Cruz \\ Embrapa Tabuleiros Costeiros \\ Avenida Beira Mar 3250 Jardins CEP:49025-040 - Aracaju-SE, Brasil \\ mascruz@cpatc.embrapa.br
}

\begin{abstract}
The characterization of basins is an important step of hydrologic modeling, considering correct representation of land covering and land use, morphology and climate variables. Geotechnologies allow obtaining this kind of information with good accuracy in short time periods. Geographic Information Systems (GIS) produce representative maps of useful variables, organizing spatial data to decision makers in water resources management. This study aimed showing the data organization from a project in Japaratuba river basin in Sergipe state, trying to illustrate the great potential of GIS in hydrographic systems and climate variables characterization.
\end{abstract}

Palavras-chave: hydrographic basin, geotechnologies, spatial analyses, bacias hidrográficas, geotecnologias, análise espacial. 


\section{Introdução}

A bacia hidrográfica constitui-se legalmente na unidade de planejamento e gestão dos recursos hídricos no Brasil, estabelecida como o sistema "fechado" de balanço dos processos constituintes de ciclo hidrológico. Assim sendo, a avaliação das alterações sobre estes processos decorrentes de intervenções humanas no uso e cobertura do solo em uma determinada região deve considerar a bacia como o espaço geográfico de ação. Segundo Zeiler (1999) os sistemas de informação geográfica (SIGs) têm como função proporcionar uma estrutura espacial para auxiliar as tomadas de decisões para o uso racional dos recursos naturais e gerenciamento das ações antrópicas sobre estes recursos. Os SIGs constituem-se em um conjunto harmônico composto por uma base de dados, um ou mais softwares que permitam a manipulação de informações e uma interface gráfica para acesso do usuário (Câmara \& Queiroz, 2008).

A aplicação dos SIGs em estudos relacionados aos recursos hídricos tem sido crescente, principalmente pela facilidade de manipulação de mapas, organização de banco de dados e de obtenção de informações geomorfológicas das bacias hidrográficas. Assim, as ferramentas de geotecnologias têm se consolidado como meio eficaz de caracterização dos sistemas hidrográficos, possibilitando, por exemplo, a determinação de valores para variáveis de cobertura e uso do solo, variabilidade espacial de parâmetros climáticos, identificação de fontes poluidoras significativas e o mapeamento de áreas vulneráveis a processos erosivos.

As macrobacias hidrográficas no estado de Sergipe apresentam-se em diferentes estágios de degradação resultantes de atividades agrícolas, extrativistas, urbanas e industriais, que promovem alterações significativas nos processos hidrológicos e na qualidade das águas dos rios do Estado. A caracterização morfométrica destas bacias associada aos usos e cobertura da terra preponderantes, constituem-se em pré-requisitos indispensáveis para o adequado processo de gestão dos recursos hídricos e planejamento de estratégias de manejo dos solos. Nestas atividades a aplicação de geotecnologias vem representando ganhos significativos de tempo e economia de recursos públicos.

A bacia do rio Japaratuba mostra-se como singular nesta situação, pois apresenta o rio principal de mesmo nome, com processos de degradação avançados em alguns trechos, resultado de avanços históricos nas atividades agropecuárias, de extração mineral e despejos industriais sem o devido controle. Esta bacia possui uma importância estratégica para o estado de Sergipe, pois abriga o maior campo petrolífero terrestre do país, o campo de Carmópolis, com mais de $150 \mathrm{~km}^{2}$ e 1.200 poços, que faz uso da porção inferior do rio Japaratuba para o despejo das águas residuárias do processo de exploração, causando alterações significativas na biota do rio, resultado da presença de metais na água e no sedimento. Assim, este estudo apresenta resultados do processo de caracterização física e de variáveis hidrológicas na bacia a partir do uso de geotecnologias, com fins de fornecer subsídios para modelagens matemáticas de cenários de intervenção.

\section{Materiais e Método}

O estudo foi desenvolvido na bacia do rio Japaratuba em Sergipe, que se constitui na menor bacia principal em extensão territorial, com cerca de $1.700 \mathrm{~km}^{2}$, o que representa aproximadamente $7,8 \%$ da área do Estado de Sergipe e é totalmente contida pelos limites deste. O rio Japaratuba tem aproximadamente $92 \mathrm{~km}$ de extensão, nasce na Serra da Boa Vista, na divisa entre os municípios de Feira Nova e Graccho Cardoso, e deságua no Oceano Atlântico, no município de Pirambu. A vazão média do rio Japaratuba é da ordem de 11,0

$\mathrm{m}^{3} / \mathrm{s}$ e a mínima $\left(\mathrm{Q}_{7,10}\right)$ de $0,22 \mathrm{~m}^{3} / \mathrm{s}$, o que demonstra o baixo potencial hídrico da bacia, caracterizando-a como uma bacia com deficiência hídrica (JICA/SEPLANTEC, 2000). Seus 
principais afluentes são os rios Japaratuba-Mirim, pela margem esquerda, e Siriri, pela direita. A população residente na área da bacia é de aproximadamente 92.200 habitantes, o que representa 5,6\% da população do Estado, dos quais cerca de $61 \%$ moram nas parcelas urbanas de municípios como Capela, Japaratuba, Carmópolis, Rosário do Catete e Siriri, entre outros. O limite noroeste da bacia é atingido pelo Polígono das Secas, abrangendo cerca de $13 \%$ da área total da bacia. A precipitação na bacia apresenta valores anuais médios de $1.270 \mathrm{~mm}$, com cerca de $900 \mathrm{~mm} /$ ano na sua porção extrema noroeste e $1.500 \mathrm{~mm} /$ ano junto à sua foz. Apesar da sua baixa disponibilidade hídrica, os usos múltiplos da água superficial apresentam-se intensos na bacia, destacando-se a utilização da água nas atividades de exploração mineral, principalmente de petróleo/gás e potássio, abastecimento humano e irrigação. Alterações significativas na qualidade da água do rio Japaratuba e afluentes vêm sendo verificadas ao longo dos anos, constituindo elementos de estudos diversos, que apontam para causas associadas a alterações de cobertura do solo e despejos de efluentes das atividades humanas. Segundo Pantaleão (2006), a exploração de petróleo na bacia, que se constitui no maior campo petrolífero terrestre do país, o campo de Carmópolis, com mais de $150 \mathrm{~km}^{2}$ e 1.200 poços, faz uso da porção inferior do rio Japaratuba para o despejo das águas residuárias do processo de exploração, causando alterações significativas na biota do rio, resultado da presença de metais na água e no sedimento. A poluição das águas por efluentes urbanos mostra-se presente nas proximidades dos municípios de Japaratuba, Capela e Nossa Senhora das Dores, com predominância de esgotos domésticos não tratados. O uso agrícola na bacia concentra-se na produção de cana-de-açúcar próxima a planície aluvial do rio e em pastagens, estas espalhadas ao longo de toda a bacia. Estas atividades têm provocado a remoção significativa da mata ciliar, contribuindo para a aceleração dos processos erosivos e para o assoreamento dos leitos dos rios. A mata ciliar no rio Japaratuba encontra-se atualmente reduzida a aproximadamente $1 \%$ do que a legislação ambiental determina como adequado, chegando a 0,14\% no seu principal afluente, o rio Japaratuba-Mirim (Rocha et al., 2009).

A aplicação de ferramentas de geotecnologias foi realizada por meio do software ArcGIS (ESRI, 2009) para caracterizar a bacia fisicamente, utilizando funções do módulo Spatial Analyst - Hydrology e quanto à distribuição espacial da chuva, por meio do módulo Geostatistical Analyst. A partir do Modelo Digital de Elevação para a bacia completa, obtido a partir de dados SRTM (Shuttle Radar Topography Mission) segundo produtos refinados por Miranda (2005), foram extraídas informações úteis para os processos de simulação hidrológica, tais como altitudes máximas, médias e mínimas; limites e área total da bacia; comprimentos de talvegues; declividades médias e direções de fluxo. Foram gerados também mapas de distribuição espacial das precipitações anuais e mensais que ocorrem na bacia a partir do uso de geoestatística. Para tanto foram utilizados dados consistidos de 15 postos pluviográficos/métricos que dispunham de séries históricas com mais de 15 anos de monitoramento contínuo.

\section{Resultados}

A partir de dados SRTM (resolução 90m) pós-processados por Miranda (2005) foi realizada a delimitação da bacia do rio Japaratuba por meio do módulo Hydrology presente na extensão Spatial Analyst do ArcGIS. Utilizando um recorte da cena SC-24-Z-B, foi realizado o preenchimento de imperfeições (Comando Fill) e gerado o mapa de direção de fluxo (Comando Flow Direction), que são apresentados nas Figuras 1A e 1B. A partir do mapa de direção de fluxo produziu-se o mapa de fluxo acumulado (Comando Flow Accumulation) (Figura 1C) e, na sequiência, foram definidas as bacias contribuintes (Comando Basin) (Figura 1D). Observa-se na Figura 1D na cor azul ao centro, a bacia do rio Japaratuba. 


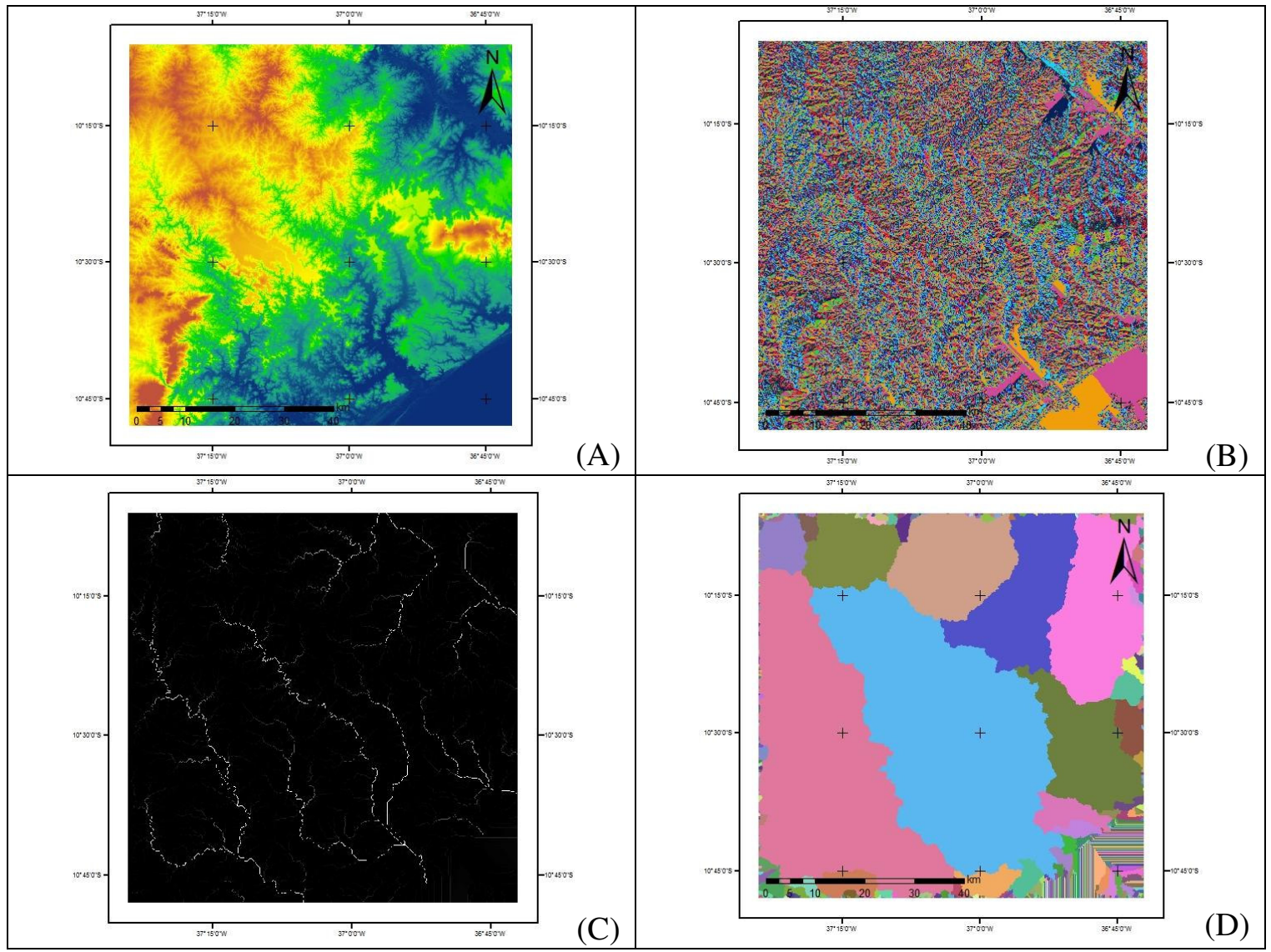

Figura 1 - Processo de determinação dos limites da bacia do rio Japaratuba a partir do Modelo Digital de Elevação. (A) - Recorte da cena SC-24-Z-B obtida de SRTM (Miranda, 2005); (B) Mapa de direção de fluxo; (C) Mapa de Fluxo Acumulado; (D) Bacias hidrográficas delimitadas.

A partir da imagem raster produzida, extraiu-se o polígono correspondente aos limites da bacia do rio Japaratuba, e utilizou-se deste para promover um recorte no MDT, produzindo o mapa da Figura 2. Observa-se que a amplitude de variação da altitude na bacia é de $288,0 \mathrm{~m}$, com a cota máxima situada a $289,0 \mathrm{~m}$ e sua foz praticamente ao nível do mar. O mapa apresentado permite a obtenção de várias informações físicas sobre a bacia, que são apresentadas na Tabela 1. A informação morfométrica produzida a partir do MDT possibilita a determinação, por exemplo, da declividade média da bacia, que se situa na ordem de $0,4 \%$, parâmetro este essencial na estimativa do tempo de concentração e tempos de resposta de diferentes partes da bacia para simulação do escoamento superficial decorrente de eventos de precipitação. 


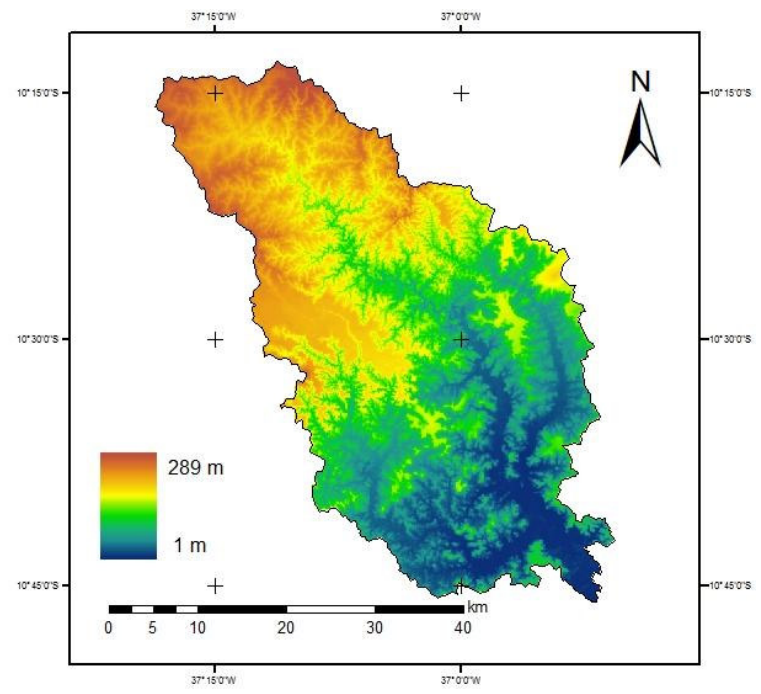

Figura 2 - Modelo digital do terreno para a bacia do rio Japaratuba em Sergipe.

Tabela 1 - Características da bacia do rio Japaratuba obtidas por meio do MDT

\begin{tabular}{|c|c|c|c|c|}
\hline Área $\left(\mathrm{km}^{2}\right)$ & $\begin{array}{c}\text { Amplitude } \\
\text { altimétrica }(\mathrm{m})\end{array}$ & $\begin{array}{c}\text { Comprimento } \\
\text { bacia }(\mathrm{km})\end{array}$ & $\begin{array}{c}\text { Comprimento } \\
\text { rio principal } \\
(\mathrm{km})\end{array}$ & $\begin{array}{c}\text { Declividade } \\
\text { média }(\mathrm{m} / \mathrm{m})\end{array}$ \\
\hline $1.653,6$ & 288,0 & 71,4 & 94,1 & 0,0040 \\
\hline
\end{tabular}

A análise da distribuição espacial das precipitações na bacia foi realizada com o uso de geotecnologias, buscando representar a variabilidade espacial para efeitos de simulação matemática na bacia com modelos chuva-vazão. Foram utilizados dados de 15 postos de registro diário de precipitação com séries históricas superiores a 10 anos, obtidos através do portal Hidroweb (ANA, 2009). A Figura 3 apresenta a bacia do rio Japaratuba e os postos de chuva localizados no interior da bacia ou próximos aos seus limites. A Tabela 2 resume as características dos postos selecionados.

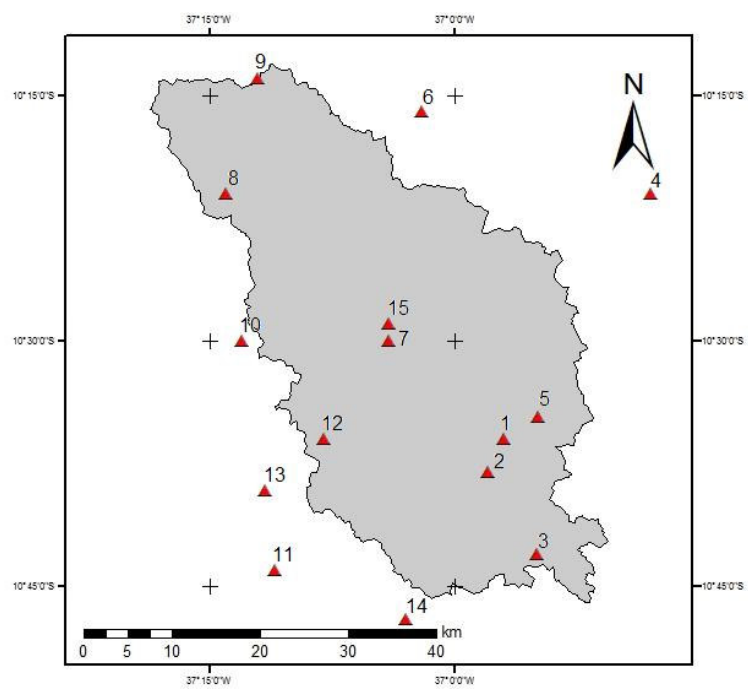

Figura 3 - Postos com registros de precipitação para a bacia do rio Japaratuba 
Tabela 2 - Precipitações médias anuais e mensais para os postos na bacia do rio Japaratuba

\begin{tabular}{|c|c|c|c|c|c|c|c|c|c|c|}
\hline Num & 1 & 2 & 3 & \multicolumn{2}{|c|}{4} & \multicolumn{2}{|c|}{5} & 6 & 7 & 8 \\
\hline POSTO & 1036014 & 1036020 & 1036033 & \multicolumn{2}{|c|}{1036054} & \multicolumn{2}{|c|}{1036063} & 1037003 & 1037009 & 1037013 \\
\hline LATI & $-10,600$ & $-10,633$ & $-10,717$ & \multicolumn{2}{|c|}{$-10,350$} & \multicolumn{2}{|c|}{$-10,578$} & $-10,267$ & $-10,500$ & $-10,350$ \\
\hline LONG & $-36,950$ & $-36,967$ & $-36,917$ & \multicolumn{2}{|c|}{$-36,800$} & \multicolumn{2}{|c|}{$-36,916$} & $-37,033$ & $-37,067$ & $-37,233$ \\
\hline JAN & 36,2 & 37,5 & 38,5 & \multicolumn{2}{|c|}{41,8} & \multicolumn{2}{|c|}{43,4} & 31,7 & 43,5 & 30,2 \\
\hline FEV & 48,6 & 90,0 & 77,5 & \multicolumn{2}{|c|}{62,6} & \multicolumn{2}{|c|}{40,1} & 37,5 & 52,6 & 41,8 \\
\hline MAR & 85,8 & 109,9 & 125,3 & \multicolumn{2}{|c|}{107,1} & \multicolumn{2}{|c|}{59,1} & 69,1 & 119,6 & 73,8 \\
\hline ABR & 171,4 & 196,3 & 242,5 & \multicolumn{2}{|c|}{186,9} & \multicolumn{2}{|c|}{172,7} & 98,0 & 177,5 & 102,7 \\
\hline MAI & 249,1 & 288,1 & 297,5 & \multicolumn{2}{|c|}{153,6} & \multicolumn{2}{|c|}{228,3} & 149,7 & 225,7 & 133,6 \\
\hline JUN & 181,0 & 246,7 & 212,6 & & 0 & 28 & & 146,0 & 197,3 & 111,5 \\
\hline JUL & 185,7 & 217,2 & 203,2 & & 5,8 & $20 t$ & & 140,9 & 177,6 & 115,9 \\
\hline AGO & 118,4 & 151,9 & 124,1 & & & 13 & & 88,7 & 131,7 & 75,5 \\
\hline SET & 69,2 & 106,3 & 103,5 & & & 68 & & 60,3 & 74,1 & 51,0 \\
\hline OUT & 53,6 & 63,4 & 59,3 & & 6 & 78 & & 34,0 & 34,9 & 23,7 \\
\hline NOV & 38,0 & 40,9 & 43,1 & & & 47 & & 36,2 & 35,2 & 14,5 \\
\hline DEZ & 33,8 & 24,6 & 40,4 & & ,7 & 36 & & 27,7 & 31,0 & 23,6 \\
\hline ANUAL & 1270,9 & 1572,7 & 1567,4 & & 4,7 & 140 & & 919,8 & 1300,6 & 797,8 \\
\hline Num & 9 & 10 & 11 & & & & & 13 & 14 & 15 \\
\hline POSTO & 1037016 & 1037036 & 10370 & & 103 & 047 & & 37049 & 1037050 & 1037078 \\
\hline LATI & $-10,233$ & $-10,500$ & $-10,7$ & & & 600 & & 0,653 & $-10,783$ & $-10,483$ \\
\hline LONG & $-37,200$ & $-37,217$ & $-37,1 \varepsilon$ & & & 133 & & 7,193 & $-37,050$ & $-37,067$ \\
\hline JAN & 37,5 & 36,1 & 51,0 & & & & & 5,4 & 37,0 & 49,3 \\
\hline FEV & 39,1 & 43,7 & 79,0 & & & & & 6,9 & 74,0 & 41,7 \\
\hline MAR & 66,6 & 78,4 & 99,4 & & & & & 1,6 & 96,1 & 69,4 \\
\hline ABR & 101,6 & 118,2 & 178, & & & & & 73,8 & 212,1 & 159,9 \\
\hline MAI & 131,6 & 188,2 & 263 & & & & & 22,1 & 270,1 & 197,1 \\
\hline JUN & 115,7 & 160,9 & 287, & & & & & 26,6 & 189,3 & 208,1 \\
\hline JUL & 119,0 & 152,0 & 235 & & & & & 98,0 & 181,3 & 182,9 \\
\hline AGO & 74,0 & 100,4 & 174, & & & & & 24,9 & 120,5 & 113,1 \\
\hline SET & 49,2 & 59,2 & 120 & & & & & 2,1 & 71,8 & 78,5 \\
\hline OUT & 21,0 & 40,6 & 53,4 & & & & & 4,6 & 36,1 & 54,5 \\
\hline NOV & 18,8 & 37,3 & 49,8 & & & & & 1,2 & 41,6 & 45,2 \\
\hline DEZ & 16,3 & 35,6 & 35,1 & & & & & 8,3 & 20,5 & 30,2 \\
\hline ANUAL & 790,5 & 1050,5 & 1627 & & & & & 45,5 & 1350,4 & 1229,9 \\
\hline
\end{tabular}

Observa-se que as chuvas anuais e mensais têm grande variação em função da localização dos postos, uma vez que a bacia transpassa diferentes regiões climáticas. Considerando meses chuvosos, como maio, por exemplo, observa-se um valor médio de $215,0 \mathrm{~mm}$, no entanto têm-se registros variando de 131,6 mm a 297,4 mm, a depender da posição espacial do posto. Para as precipitações anuais, as alturas pluviométricas variaram de 790,5 $\mathrm{mm}$ a 1627,9 mm, com um valor médio de $1227,9 \mathrm{~mm}$ e desvio padrão de 273,9 $\mathrm{mm}$. Esta simples análise reforça a necessidade da consideração da localização espacial das fontes dos dados na interpretação e aplicação das informações produzidas.

Utilizando a extensão Geostatistical Analyst do ArcGIS, foram gerados semivariogramas experimentais e avaliada a existência de dependência espacial para as precipitações anuais e mensais. Após, foram produzidos mapas através da krigeagem ordinária que permitam a obtenção da informação para áreas da bacia sem cobertura por postos. A Figura 4 apresenta alguns exemplos dos produtos obtidos. Observa-se em todos os casos uma redução nas lâminas precipitadas do litoral (foz da bacia) para o interior, representando bem a transição 
presente na bacia de clima litorâneo, passando pelo agreste e chegando ao sertão sergipano. Tais mapas permitem a melhor distribuição das precipitações pontuais registradas em aparelhos dentro da bacia, possibilitando uma interpretação mais próxima da realidade quando da simulação com modelos hidrológicos.

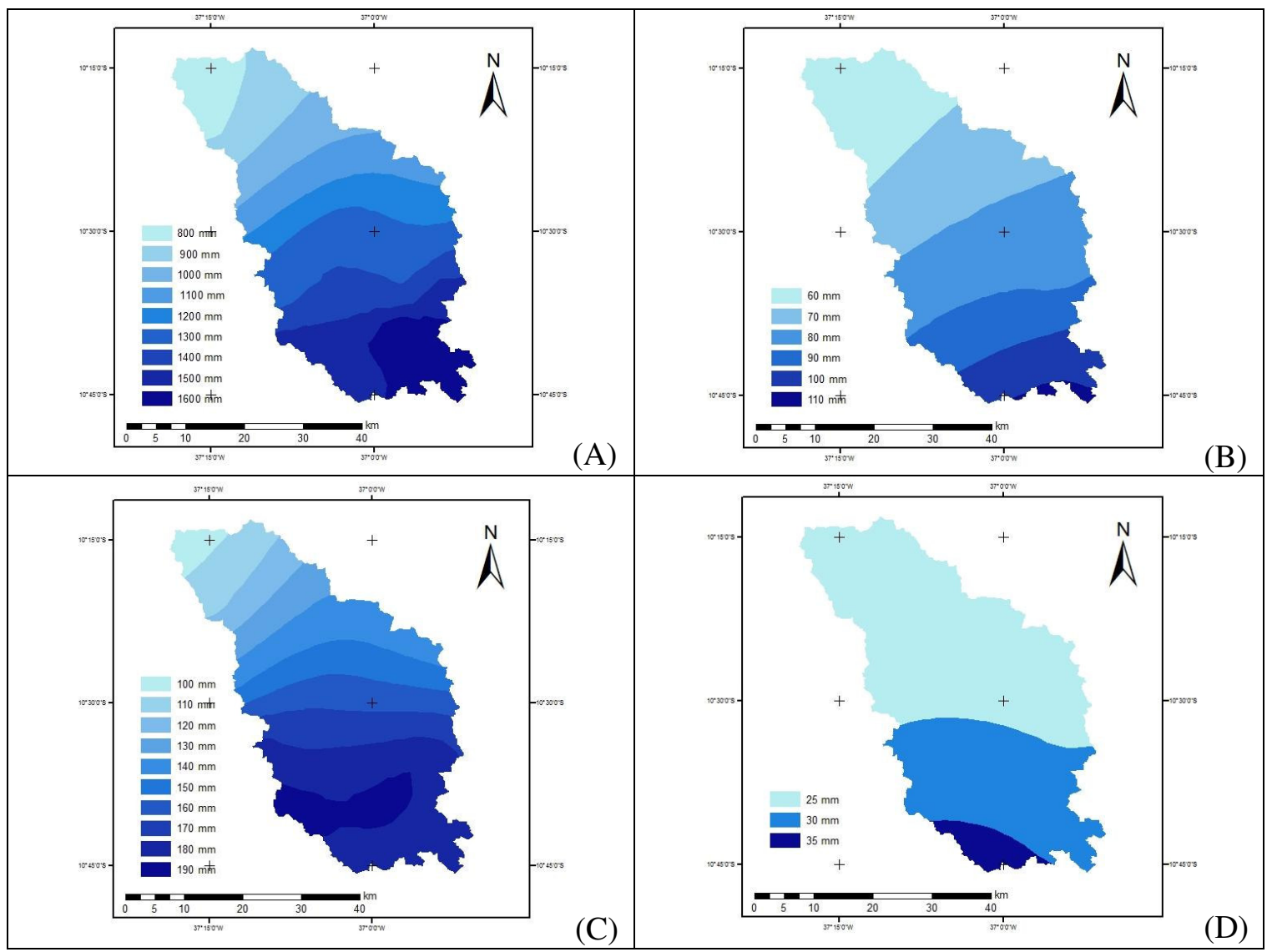

Figura 4 - Mapas de distribuição espacial de precipitações anuais e mensais na bacia do rio Japaratuba em Sergipe - (A) Precipitação média anual; (B) média do mês de março; (C) média do mês de julho; (D) média do mês de novembro.

\section{Conclusões}

1. A aplicação de geotecnologias permitiu de forma precisa e eficaz a caracterização física e da variação espacial de precipitações médias na bacia do rio Japaratuba;

2. Há um grande potencial de exploração das imagens SRTM na caracterização morfométrica de bacias hidrográficas;

3. O uso de técnicas de geoestatística, como a krigeagem, possibilitam, desde que as variáveis possuam correlação espacial, a produção de informações em regiões sem dados.

\section{Referências bibliográficas}

ANA. HidroWeb. Sistema de informações Hidrológicas. <http://hidroweb.ana.gov.br/>. Acesso em: 29 ago. 2009. 
CÂMARA, G.; QUEIROZ, G.R. Arquitetura de sistemas de informações geográficas. In: CÂMARA, G.; DAVIS, C.; MONTEIRO, A.M.V. Introdução à ciência da geoinformação. Disponível em: <http://www.dpi.inpe.br/gilberto/livro/introd/>. Acesso em: 4 jul. 2008.

ESRI. ArcGIS desktop help. v. 9.1. Disponível em: <http://webhelp.esri.com/arcgisdesktop /9.1/index.cfm>. Acesso em: 20 jun. 2009.

JICA/SEPLANTEC. Estudo sobre o Desenvolvimento dos Recursos Hídricos no Estado de Sergipe na República Federativa do Brasil. Japan International Cooperation Agency. Secretaria de Estado do Planejamento e da Ciência e Tecnologia. Aracaju. 2000.

PANTALEÃO, S.M. Impacto genotóxico de poluentes químicos presentes na água e sedimentos do rio Japaratuba. 2006. 107f. Tese (Doutorado) - Programa de Pós-Graduação em Genética e Bioquímica, Universidade Federal de Uberlândia. Uberlândia, 2006.

ROCHA, J.C.S., CORREIA, C.O., ROCHA, C.L. Bacia hidrográfica do rio Japaratuba: uma visão da gestão de recursos hídricos frente ao uso e ocupação da terra. In: ENCONTRO DE RECURSOS HÍDRICOS EM SERGIPE. 2., 2009, Aracaju. Anais... Aracaju: Embrapa Tabuleiros Costeiros. 2009.

MIRANDA, E. E. de; (Coord.). Brasil em Relevo. Campinas: Embrapa Monitoramento por Satélite, 2005. Disponível em: <http://www.relevobr.cnpm.embrapa.br>. Acesso em: 27 ago. 2009.

ZEILER, M. Modeling our real world - The ESRI guide to geodatabase design. Califórnia: Published by Environmental Systems Research Institute, 1999. 202p. 